\title{
SPECTRAL THEORY OF ELLIPTIC OPERATORS IN EXTERIOR DOMAINS
}

\author{
FRITZ GESZTESY AND MARK M. MALAMUD
}

\begin{abstract}
We consider various closed (and self-adjoint) extensions of elliptic differential expressions of the type $\mathcal{A}=\sum_{0 \leqslant|\alpha|,|\beta| \leqslant m}(-1)^{\alpha} D^{\alpha} a_{\alpha, \beta}(x) D^{\beta}, a_{\alpha, \beta}(\cdot) \in C^{\infty}(\bar{\Omega})$, on smooth (bounded or unbounded) domains $\Omega$ in $\mathbb{R}^{n}$ with compact boundary $\partial \Omega$. Using the concept of boundary triples and operator-valued Weyl-Titchmarsh functions, we prove various trace ideal properties of powers of resolvent differences of these closed realizations of $\mathcal{A}$ and derive estimates on eigenvalues of certain self-adjoint realizations in spectral gaps of the Dirichlet realization.

Our results extend classical theorems due to Višik, Povzner, Birman, and Grubb.
\end{abstract}

\section{INTRODUCTION}

Let $\Omega$ be an open domain in $\mathbb{R}^{n}$ (bounded or unbounded) with compact boundary $\partial \Omega$. Throughout we assume that $\partial \Omega$ is an $(n-1)$-dimensional (not necessarily connected) $C^{\infty}$-manifold. Let $\mathcal{A}$ be the differential expression

$$
\mathcal{A}=\sum_{0 \leqslant|\alpha|,|\beta| \leqslant m}(-1)^{\alpha} D^{\alpha} a_{\alpha, \beta}(x) D^{\beta}, \quad a_{\alpha, \beta}(\cdot) \in C^{\infty}(\bar{\Omega}),
$$

$\operatorname{ord}(\mathcal{A})=2 m$, which is elliptic in $\bar{\Omega}$. Moreover, we assume that $\mathcal{A}$ is properly elliptic in $\bar{\Omega}$ (which is automatically satisfied if either $n>2$ or the symbol of $\mathcal{A}$ is real, cf. [11]). In addition to (1.1) we consider its formal adjoint $\mathcal{A}^{\top}=\sum_{0 \leqslant|\alpha|,|\beta| \leqslant m}(-1)^{\alpha} D^{\beta} \overline{a_{\beta, \alpha}(x)} D^{\beta}$, which is also properly elliptic in $\bar{\Omega}$ (cf. [11]).

Denote by $A=A_{\min }\left(A^{\top}=A_{\min }^{\top}\right)$ the minimal operator associated in $L^{2}(\Omega)$ with the differential expression $\mathcal{A}$ (resp., $\mathcal{A}^{\top}$ ), that is, the closure of $\mathcal{A}$ defined on $C_{0}^{\infty}(\Omega)$. The maximal operators $A_{\max }$ and $A_{\max }^{\top}$ are then defined by $A_{\max }=\left(A_{\min }^{\top}\right)^{*}=\left(A^{\top}\right)^{*}$ and $A_{\max }^{\top}=\left(A_{\min }\right)^{*}=A^{*}$, respectively. We emphasize that $H^{2 m}(\Omega) \subset \operatorname{dom}\left(A_{\max }\right) \subset H_{\text {loc }}^{2 m}(\Omega)$, while $\operatorname{dom}\left(A_{\max }\right) \neq H^{2 m}(\Omega)$.

After the pioneering work by Vishik [15], nonlocal boundary value problems of the form $A_{\max } u=f,(\partial u / \partial n-$ $K u) \uparrow \partial \Omega=0$ for elliptic operators (1.1) (with $m=1$ ) in bounded domains were considered by numerous authors (see, e.g., [3, 9] and the references therein). Vishik was the first to consider these problems in the framework of extension theory of dual pairs of operators. Starting with a formula for the domain $\operatorname{dom}\left(A_{\max }\right)$ of $A_{\max }$, he applied it to an appropriate regularization of the classical Green's formula, using the Calderon operator. The latter allowed him to extend the Green's formula from $H^{2 m}(\Omega)$ to $\operatorname{dom}\left(A_{\max }\right)$. The next fundamental contribution to the subject was made by Grubb [9]. Using the theory of Lions and Magenes [11], Grubb substantially extended and completed the results of [15]. In particular, Grubb obtained the (regularized) Green's formula which (in the special case $m=1$ ) reads as follows:

$$
\left(A_{\max } u, v\right)_{L^{2}(\Omega)}-\left(u, A_{\max }^{\top} v\right)_{L^{2}(\Omega)}=\left(\widetilde{\Gamma}_{\Omega, 1} u, \widetilde{\Gamma}_{\Omega, 0}^{\top} v\right)_{1 / 2,-1 / 2}-\left(\widetilde{\Gamma}_{\Omega, 0} u, \widetilde{\Gamma}_{\Omega, 1}^{\top} v\right)_{-1 / 2,1 / 2} .
$$

Here $(\cdot, \cdot)_{s,-s}$ denotes the duality pairing between $H^{s}(\partial \Omega)$ and $H^{-s}(\partial \Omega), u \in \operatorname{dom}\left(A_{\max }\right), v \in \operatorname{dom}\left(A_{\max }^{\top}\right)$, and $\widetilde{\Gamma}_{\Omega, 0}, \widetilde{\Gamma}_{\Omega, 1}, \Gamma_{\Omega, 0}^{\top}$, and $\widetilde{\Gamma}_{\Omega, 1}^{\top}$ are regularized trace operators, having the properties

$$
\widetilde{\Gamma}_{\Omega, 1}: \mathcal{D}\left(A_{\max }\right) \rightarrow H^{1 / 2}(\partial \Omega), \quad \widetilde{\Gamma}_{\Omega, 0}^{\top}: \mathcal{D}\left(A_{\max }\right) \rightarrow H^{-1 / 2}(\partial \Omega), \quad \operatorname{ran}\left(\left(\widetilde{\Gamma}_{\Omega, 1}, \widetilde{\Gamma}_{\Omega, 0}^{\top}\right)\right)=H^{1 / 2}(\partial \Omega) \times H^{-1 / 2}(\partial \Omega) .
$$

Later, we will use a somewhat different approach (cf. Proposition 2.7).

1991 Mathematics Subject Classification. Primary 35P20, 35J40, 47F05; Secondary 47A10.

Key words and phrases. Elliptic Partial Differential Equations, Spectral Theory, Boundary Triples, Weyl-Titchmarsh Operators. 
On the other hand, during the past three decades a new approach to the extension theory, based on the concept of a boundary triple and the corresponding operator-valued Weyl-Titchmarsh function, was developed in [7] (cf. the references therein for the symmetric case) and in [13] (in the case of dual pairs). In this paper we apply some results and technique from [7] and [13] to elliptic operators on unbounded domains. The most important ingredients from the elliptic theory we need are the regularized Green's formula and a priori coercivity-type estimates for the elliptic realizations $\widehat{A}_{B}$ of $\mathcal{A}$ (see (2.2) below). To obtain the latter on unbounded domains one needs additional restrictions on the coefficients of $\mathcal{A}$, since an elliptic realization is not necessarily coercive. Here we restrict ourselves to the case of bounded coefficients $a_{\alpha, \beta}(\cdot)$. Using the formalism of boundary triples and the corresponding operator-valued Weyl-Titchmarsh functions in $[7,13]$, we investigate the resolvent difference of two realizations and complement the results of Povzner [14], Birman [5], and Grubb [10] in this direction. In addition, assuming $A_{\min }>0$, we compute the number of negative eigenvalues of a realization $A_{K}$ and the number of eigenvalues of $A_{K}$ within spectral gaps of the Dirichlet realization $\widehat{A}_{\gamma_{D}}$, where $\gamma_{D}=\left\{\gamma_{j}\right\}_{0}^{m-1}$.

Notations. $\mathfrak{H}$ and $\mathcal{H}$ represent complex, separable Hilbert spaces; $\mathcal{B}(\mathcal{H}), \mathcal{B}_{\infty}(\mathcal{H})$, and $\mathcal{C}(\mathcal{H})$ denote the sets of bounded, compact, and closed linear operators in $\mathcal{H} ; \operatorname{dom}(\cdot), \operatorname{ran}(\cdot)$, and $\operatorname{ker}(\cdot)$ denote the domain, range, and kernel of a linear operator, $\rho(\cdot)$ and $\sigma(\cdot)$ stand for the resolvent set and spectrum of a linear operator. As usual, $C^{\infty}(\Omega)$ denotes the set of infinity differentiable functions in the domain $\Omega, C_{0}^{\infty}(\Omega)$ the subset of $C^{\infty}(\Omega)$-functions of compact support in $\Omega ; C_{b}(\Omega)=C(\Omega) \cap L^{\infty}(\Omega), C_{u}(\Omega)$ the set of uniformly continuous functions in $\Omega, C_{u b}(\Omega)=C_{u}(\Omega) \cap C_{b}(\Omega)$, and $H^{s}(\Omega)$ the usual Sobolev spaces.

\section{Dual pairs, boundary triples, and operator-Valued Weyl-Titchmarsh functions}

Definition 2.1. Let $A$ and $A^{\top}$ be densely defined (not necessarily closed) linear operators in $\mathfrak{H}$. Then $A$ and $A^{\top}$ form a dual pair $(D P)\left\{A, A^{\top}\right\}$ in $\mathfrak{H}$ if $(A f, g)=\left(f, A^{\top} g\right)$ for all $f \in \operatorname{dom}(A), g \in \operatorname{dom}\left(A^{\top}\right)$. An operator $\widetilde{A}$ is called a proper extension of the $D P\left\{A, A^{\top}\right\}$, and we write $\widetilde{A} \in \operatorname{Ext}\left\{A, A^{\top}\right\}$, if $A \varsubsetneqq \widetilde{A} \varsubsetneqq\left(A^{\top}\right)^{*}$.

Definition 2.2. (cf. [12], [13]) (i) Let $\mathfrak{H}, \mathcal{H}_{0}$, and $\mathcal{H}_{1}$ be complex, separable Hilbert spaces and

$$
\Gamma^{\top}=\left(\begin{array}{l}
\Gamma_{0}^{\top} \\
\Gamma_{1}^{\top}
\end{array}\right): \operatorname{dom}\left(\left(A^{\top}\right)^{*}\right) \rightarrow \mathcal{H}_{0} \oplus \mathcal{H}_{1} \text { and } \Gamma=\left(\begin{array}{c}
\Gamma_{0} \\
\Gamma_{1}
\end{array}\right): \operatorname{dom}\left(A^{*}\right) \rightarrow \mathcal{H}_{1} \oplus \mathcal{H}_{0}
$$

be linear mappings. Then $\Pi=\left\{\mathcal{H}_{0} \oplus \mathcal{H}_{1}, \Gamma^{\top}, \Gamma\right\}$ is called a boundary triple for the dual pair $\left\{A, A^{\top}\right\}$ if $\Gamma^{\top}$ and $\Gamma$ are surjective and the Green's identity holds,

$$
\left(\left(A^{\top}\right)^{*} f, g\right)_{\mathfrak{H}}-\left(f, A^{*} g\right)_{\mathfrak{H}}=\left(\Gamma_{1}^{\top} f, \Gamma_{0} g\right)_{\mathcal{H}_{1}}-\left(\Gamma_{0}^{\top} f, \Gamma_{1} g\right)_{\mathcal{H}_{0}}, \quad f \in \operatorname{dom}\left(\left(A^{\top}\right)^{*}\right), g \in \operatorname{dom}\left(A^{*}\right) .
$$

We set $A_{0}=\left(A^{\top}\right)^{*} \uparrow \operatorname{ker}\left(\Gamma_{0}^{\top}\right)$ and $A_{0}^{\top}=A^{*} \uparrow \operatorname{ker}\left(\Gamma_{0}\right)$.

(ii) The operator-valued function $M_{\Pi}(z)$ defined by

$$
\Gamma_{1}^{\top} f_{z}=M_{\Pi}(z) \Gamma_{0}^{\top} f_{z}, \quad f_{z} \in \operatorname{ker}\left(\left(A^{\top}\right)^{*}-z\right), \quad z \in \rho\left(A_{0}\right),
$$

is called the Weyl-Titchmarsh function corresponding to the boundary triple $\Pi$.

Due to Green's identity, $\left(A_{\min } u, v\right)_{L^{2}(\Omega)}=\left(u, A_{\min }^{\top} v\right)_{L^{2}(\Omega)}, u, v \in C_{0}^{\infty}(\Omega)$, the operators $A$ and $A^{\top}$ form a dual pair $\left\{A, A^{\top}\right\}$ of elliptic operators in $L^{2}(\Omega)$. Any proper extension $\widetilde{A} \in \operatorname{Ext}\left\{A, A^{\top}\right\}$ of $\left\{A, A^{\top}\right\}$ is called a realization of $\mathcal{A}$. Clearly, any realization $\widetilde{A}$ of $\mathcal{A}$ is closable. We equip $\operatorname{dom}\left(A_{\max }\right)$ and $\operatorname{dom}\left(A_{\max }^{\top}\right)$ with the corresponding graph norms. It is known (cf. $[4,11])$ that if a domain $\Omega$ is bounded, then $\operatorname{dom}\left(A_{\min }\right)=$ $\operatorname{dom}\left(A_{\min }^{\top}\right)=H_{0}^{2 m}(\Omega)$, where the norms in $\operatorname{dom}\left(A_{\text {min }}\right)$ and $H_{0}^{2 m}(\Omega)$ are equivalent. Denote by $\gamma_{j}$ the mappings $\gamma_{j}: C^{\infty}(\bar{\Omega}) \rightarrow C^{\infty}(\partial \Omega), \gamma_{j} u=\gamma_{0}\left(\partial^{j} u / \partial n^{j}\right)=\partial^{j} u / \partial n^{j}\left\lceil\partial \Omega, 1 \leqslant j \leqslant m-1, \gamma_{0} u=u\lceil\partial \Omega\right.$, where $n$ stands for the interior normal to $\partial \Omega$. Next we introduce the boundary operators $B_{j}$ as

$$
B_{j} u=\sum_{0 \leqslant|\beta| \leqslant m_{j}} b_{j \beta} \gamma_{0}\left(D^{\beta} u\right), \quad b_{j \beta}(\cdot) \in C^{\infty}(\partial \Omega), \quad \operatorname{ord}\left(B_{j}\right)=m_{j} \leqslant 2 m-1 .
$$

Here $B_{j}: C^{\infty}(\bar{\Omega}) \rightarrow C^{\infty}(\partial \Omega)$ will eventually be extended to appropriate Sobolev spaces $H^{s}(\Omega)$ and in some cases to $\mathcal{D}\left(A_{\max }\right) . B_{j}$ in $(2.1)$ can also be rewritten as $B_{j} u=b_{j} \gamma_{m_{j}} u+\sum_{0 \leqslant k \leqslant m_{j}-1} T_{j, k} \gamma_{k} u$, where $b_{j}(\cdot) \in C^{\infty}(\partial \Omega)$ and $T_{j, k}$ are tangential differential operators in $\partial \Omega$ of orders $\operatorname{ord}\left(T_{j, k}\right) \leqslant m_{j}-k$ with $C^{\infty}(\partial \Omega)$-coefficients. 
With any elliptic operator $A(1.1)$ and a system $B=\left\{B_{j}\right\}_{j=1}^{m-1}$ we associate the operator $\widehat{A}_{B}$ defined by

$$
\widehat{A}_{B}=A_{\max }\left\lceil\operatorname{dom}\left(\widehat{A}_{B}\right), \quad \operatorname{dom}\left(\widehat{A}_{B}\right)=H_{B}^{2 m}(\Omega)=\left\{u \in H^{2 m}(\Omega) \mid B u=0\right\} .\right.
$$

Our considerations are based on [11, Thm. 2.2.1]. According to this result, for any elliptic differential expression $\mathcal{A}$ in (1.1) and any normal system $\left\{B_{j}\right\}_{j=0}^{m-1}$ on $\partial \Omega$ given by (2.1), there exists a system of boundary operators $\left\{C_{j}\right\}_{j=0}^{m-1}, \operatorname{ord}\left(C_{j}\right)=\mu_{j} \leqslant 2 m-1$, such that the system $\left\{B_{0}, \ldots, B_{m-1}, C_{0}, \ldots, C_{m-1}\right\}$ is a Dirichlet system of order $2 m$ and another Dirichlet system of boundary operators $\left\{B_{j}^{\top}\right\}_{j=0}^{m-1} \cup\left\{C_{j}^{\top}\right\}_{j=0}^{m-1}$, such that the following Green's formula hold

$$
(\mathcal{A} u, v)_{L^{2}(\Omega)}-\left(u, \mathcal{A}^{\top} v\right)_{L^{2}(\Omega)}=\sum_{0 \leqslant l \leqslant m-1}\left[\left(C_{j} u, B_{j}^{\top} v\right)_{L^{2}(\partial \Omega)}-\left(B_{j} u, C_{j}^{\top} v\right)_{L^{2}(\partial \Omega)}\right], \quad u, v \in H^{2 m}(\Omega) .
$$

Next, following [9] and [11], we introduce the spaces $D_{A}^{s}(\Omega)=\left\{u \in H^{s}(\Omega) \mid A u \in H^{0}(\Omega)\right\}, s \in \mathbb{R}$, provided with the graph norm $\|u\|_{D_{A}^{s}(\Omega)}=\left(\|u\|_{s}^{2}+\|A u\|_{0}^{2}\right)^{1 / 2}$. Clearly, $D_{A}^{0}(\Omega)=\operatorname{dom}(A)$ and $D_{A}^{s}(\Omega) \hookrightarrow H^{s}(\Omega)$.

Definition 2.3. (i) The operator $\widehat{A}_{B}$ defined by (2.2) is called elliptic and is put in the class $\operatorname{Ell}(A)$ if $A$ is properly elliptic on $\Omega$ and the system $\left\{B_{j}\right\}_{j=0}^{m-1}$ is normal and satisfies the covering condition (cf. [11, Sects 2.1.1-2.1.4]) at any point of the boundary $\partial \Omega$.

(ii) The operator $\widehat{A}_{B}$ is called coercive in $H^{s}(\Omega)$ with $s \geqslant 2 m$ if the a priori estimate (2.25) in [2] (cf. also [11, Sect. 2.9.6]) holds.

We note that $\widehat{A}_{B}$ is a closed operator if $B$ satisfies the covering condition (cf. [2, Sect. 6.5], [11, Thm. 2.8.4]). If $\Omega$ is bounded, then any elliptic differential expression $\mathcal{A}$ with $C(\bar{\Omega})$-coefficients is uniformly elliptic in $\bar{\Omega}$. In this case, $\widehat{A}_{B} \in \operatorname{Ell}(A)$ if and only if $\widehat{A}_{B}$ is coercive in $H^{2 m}(\Omega)$ (see [1], [11, Sect. 2.9.6]). If $\Omega$ is unbounded, then the condition $\widehat{A}_{B} \in \operatorname{Ell}(A)$ is still necessary for coerciveness in $H^{2 m}(\Omega)$, though, it is no longer sufficient without additional assumptions on $A$.

Hypothesis 2.4. Assume that $\mathcal{A}$ is a uniformly elliptic operator, $a_{\alpha, \beta}(\cdot) \in C_{b}(\Omega)$ for $|\alpha|+|\beta| \leqslant 2 m$ and $a_{\alpha, \beta}(\cdot) \in C_{u b}(\Omega)$ for $|\alpha|+|\beta|=2 m$.

Proposition 2.5. Assume Hypothesis 2.4, $\widehat{A}_{B} \in \operatorname{Ell}(A)$, and $0 \in \rho\left(\widehat{A}_{B}\right)$. Then for any $s \in \mathbb{R}$, the mappings $B$ and $B^{\top}$ isomorphically map $Z_{A}^{s}(\Omega)=\left\{u \in D_{A}^{s}(\Omega) \mid A_{\max } u=0\right\}$ and $Z_{A^{\top}}^{s}(\Omega)=\left\{u \in D_{A^{\top}}^{s}(\Omega) \mid A_{\max }^{\top} u=0\right\}$ isomorphically onto $\Pi_{j=1}^{m} H^{s-m_{j}-(1 / 2)}(\partial \Omega)$ and onto $\Pi_{j=1}^{m} H^{s-2 m+\mu_{j}+(1 / 2)}(\partial \Omega)$, respectively.

Definition 2.6. $([9,15])(i)$ Under the assumptions of Proposition 2.5, let $\varphi \in \Pi_{j=0}^{m-1} H^{s-m_{j}-(1 / 2)}(\partial \Omega), s \in \mathbb{R}$. Then one defines $P(z) \varphi$ to be the unique $u \in Z_{A-z I_{L^{2}(\Omega)}}^{s}(\Omega)$ satisfying $B u=\varphi$.

(ii) The Calderon operator $\Lambda(z)$ is defined by

$$
\Lambda(z): \Pi_{j=0}^{m-1} H^{s-m_{j}-(1 / 2)}(\partial \Omega) \rightarrow \Pi_{j=0}^{m-1} H^{s-\mu_{j}-(1 / 2)}(\partial \Omega), \quad \Lambda(z) \varphi=C P(z) \varphi .
$$

(iii) Similarly, let $\psi \in \Pi_{j=0}^{m-1} H^{s-2 m+\mu_{j}+1 / 2}(\partial \Omega)$. Then $P(z)^{\top} \psi$ is defined to be the unique solution in $Z_{A^{\top}-z I_{L^{2}(\partial \Omega)}}^{s}(\Omega)$ of $B^{\top} u=\psi$ and the Calderon operator $\Lambda(z)^{\top}$ is defined as $\Lambda(z)^{\top} \psi=C^{\top} P_{z}^{\top} \psi$.

Let $\Delta_{\partial \Omega}$ be the Laplace-Beltrami operator in $L^{2}(\partial \Omega),-\Delta_{\partial \Omega, 1}=-\Delta_{\partial \Omega}+I_{L^{2}(\partial \Omega)}$. Then $-\Delta_{\partial \Omega, 1}=-\Delta_{\partial \Omega, 1}^{*} \geq$ $I_{L^{2}(\partial \Omega)}$. Moreover, $\left(-\Delta_{\partial \Omega, 1}\right)^{-s / 2}$ isomorphically maps $H^{0}(\partial \Omega)$ onto $H^{s}(\partial \Omega), s \in \mathbb{R}$. Next, we introduce the diagonal $m \times m$ operator matrices $-\Delta_{\partial \Omega, 1, m}$ and $-\Delta_{\partial \Omega, 1, \mu}$ with the $(j, j)$-th entry $\left(-\Delta_{\partial \Omega, 1}\right)^{\left(m_{j} / 2\right)+(1 / 4)}$ (resp., $\left.\left(-\Delta_{\partial \Omega, 1}\right)^{m-\left(\mu_{j} / 2\right)-(1 / 4)}\right)$.

Proposition 2.7. Assume Hypothesis 2.4, $\widehat{A}_{B} \in \operatorname{Ell}(A)$, and $0 \in \rho\left(\widehat{A}_{B}\right)$. Set

$$
\begin{aligned}
& \Gamma_{\Omega, 0} u=\left(-\Delta_{\partial \Omega, 1, m}\right)^{-1} B u, \quad \Gamma_{\Omega, 1} u=\left(-\Delta_{\partial \Omega, 1, \mu}\right)(C u-\Lambda(0) B u), \quad u \in \operatorname{dom}\left(A_{\max }\right), \\
& \Gamma_{\Omega, 0}^{\top} v=\left(-\Delta_{\partial \Omega, 1, \mu}\right)^{-1} B^{\top} v, \quad \Gamma_{\Omega, 1}^{\top} v=\left(-\Delta_{\partial \Omega, 1, m}\right)\left(C^{\top} v-\Lambda(0)^{\top} B^{\top} v\right), \quad v \in \operatorname{dom}\left(A_{\max }^{\top}\right) .
\end{aligned}
$$


Then the following holds:

(i) $\Pi=\left\{\mathcal{H}_{\partial \Omega} \oplus \mathcal{H}_{\partial \Omega}, \Gamma_{\Omega}, \Gamma_{\Omega}^{\top}\right\}$, with

$$
\mathcal{H}_{\partial \Omega}=\Pi_{j=0}^{m-1} H^{0}(\partial \Omega)=\Pi_{j=0}^{m-1} L^{2}(\partial \Omega), \quad \Gamma_{\Omega}=\left(\Gamma_{\Omega, 0}, \Gamma_{\Omega, 1}\right), \quad \Gamma_{\Omega}^{\top}=\left(\Gamma_{\Omega, 0}^{\top}, \Gamma_{\Omega, 1}^{\top}\right),
$$

forms a boundary triple for the dual pair $\left\{A, A^{\top}\right\}$ of elliptic operators in $L^{2}(\Omega)$. In particular, the following Green's formula holds

$\left(A_{\max } u, v\right)_{L^{2}(\Omega)}-\left(u, A_{\max }^{\top} v\right)_{L^{2}(\Omega)}=\left(\Gamma_{\Omega, 1} u, \Gamma_{\Omega, 0}^{\top} v\right)_{\mathcal{H}_{\partial \Omega}}-\left(\Gamma_{\Omega, 0} u, \Gamma_{\Omega, 1}^{\top} v\right)_{\mathcal{H}_{\partial \Omega}}, \quad u \in \operatorname{dom}\left(A_{\max }\right), v \in \operatorname{dom}\left(A_{\max }^{\top}\right)$.

(ii) The corresponding operator-valued Weyl-Titchmarsh function is given by

$$
M_{\Omega, \Pi}(z)=\left(-\Delta_{\partial \Omega, 1, \mu}\right)(\Lambda(z)-\Lambda(0))\left(-\Delta_{\partial \Omega, 1, m}\right), \quad z \in \rho\left(\widehat{A}_{B}\right) .
$$

In the context of operator-valued Weyl-Titchmarsh functions and elliptic partial differential operators we also refer to the recent preprint [6] (and the references cited therein).

Definition 2.8. For any operator $K: \operatorname{dom}(K) \rightarrow \Pi_{j=0}^{m-1} H^{-\mu_{j}-(1 / 2)}(\partial \Omega), \operatorname{dom}(K) \subseteq \Pi_{j=0}^{m-1} H^{-m_{j}-(1 / 2)}(\partial \Omega)$, we set

$$
A_{K}=A_{\max }\left\lceil\operatorname{dom}\left(A_{K}\right), \quad \operatorname{dom}\left(A_{K}\right)=\left\{u \in \operatorname{dom}\left(A_{\max }\right) \mid B u \in \operatorname{dom}(K), C u=K B u\right\} .\right.
$$

Definition 2.9. Define $\mathcal{S}_{p}(\mathfrak{H})=\left\{T \in \mathcal{B}_{\infty}(\mathfrak{H}) \mid s_{j}(T)=O\left(j^{-1 / p}\right)\right.$ as $\left.j \rightarrow \infty\right\}, p>0$, where $s_{j}(T), j \in \mathbb{N}$, denote the singular values of $T$ (i.e., the eigenvalues of $\left(T^{*} T\right)^{1 / 2}$ ordered in decreasing magnitude, counting multiplicity).

Theorem 2.10. Assume the conditions of Proposition 2.7 and suppose that $0 \in \rho\left(\widehat{A}_{C}\right)$ and $K \in \mathcal{C}\left(\mathcal{H}_{\partial \Omega}\right)$. Then: (i) For any realization $A_{K} \in \mathcal{C}\left(L^{2}(\Omega)\right)$ of the form (2.7), satisfying $\rho\left(A_{K}\right) \cap \rho\left(\widehat{A}_{B}\right) \neq \emptyset$, the following holds,

$$
\left[\left(A_{K}-z I_{L^{2}(\Omega)}\right)^{-l}-\left(\widehat{A}_{B}-z I_{L^{2}(\Omega)}\right)^{-l}\right] \in \mathcal{S}_{\frac{n-1}{2 m l-1 / 2}}\left(L^{2}(\Omega)\right), \quad z \in \rho\left(A_{K}\right) \cap \rho\left(\widehat{A}_{B}\right), \quad \ell \in \mathbb{N} .
$$

(ii) If $B=\left\{B_{j}\right\}_{j=0}^{m-1}$ is a Dirichlet system, $K \in \mathcal{B}\left(\mathcal{H}_{\partial \Omega}\right)$, and $\rho\left(A_{K}\right) \cap \rho\left(\widehat{A}_{B}\right) \neq \emptyset$, one has

$$
\left[\left(A_{K}-z I_{L^{2}(\Omega)}\right)^{-1}-\left(\widehat{A}_{B}-z I_{L^{2}(\Omega)}\right)^{-1}\right] \in \mathcal{S}_{\frac{n-1}{2 m}}\left(L^{2}(\Omega)\right), \quad z \in \rho\left(A_{K}\right) \cap \rho\left(\widehat{A}_{B}\right) .
$$

Combining Weyl's theorem with Theorem 2.10 one obtains the following result:

Corollary 2.11. Assume the conditions of Theorem 2.10. Then, $\sigma_{\mathrm{ess}}\left(A_{K}\right)=\sigma_{\mathrm{ess}}\left(\widehat{A}_{B}\right)$.

In the case of elliptic realizations $\widehat{A}_{G} \in \operatorname{Ell}(A)$, we have the following stronger result:

Theorem 2.12. Suppose that the conditions of Proposition 2.7 are satisfied and $\widehat{A}_{G} \in \operatorname{Ell}(A)$, that is, $\widehat{A}_{G}$ is the elliptic realization of $A$ with $G=\left\{G_{j}\right\}_{j=0}^{m-1}$. Then for any $\ell \in \mathbb{N}$,

$$
\left[\left(\widehat{A}_{G}-z I_{L^{2}(\Omega)}\right)^{-\ell}-\left(\widehat{A}_{B}-z I_{L^{2}(\Omega)}\right)^{-\ell}\right] \in \mathcal{S}_{\frac{n-1}{2 m \ell}}\left(L^{2}(\Omega)\right), \quad z \in \rho\left(\widehat{A}_{G}\right) \cap \rho\left(\widehat{A}_{B}\right) .
$$

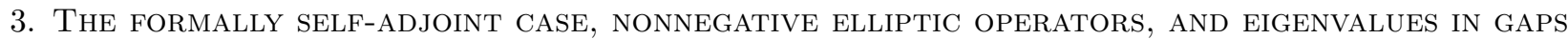

Let $\mathcal{A}$ be a formally self-adjoint elliptic differential expression of the form (1.1), that is, $\mathcal{A}=\mathcal{A}^{\top}$ or equivalently, $a_{p, q}=\overline{a_{q, p}} \in C^{\infty}(\bar{\Omega})$. In this case $A=A_{\min }=A_{\min }^{\top}=A^{\top}$, that is, $A$ is symmetric, and $A_{\max }=$ $\left(A_{\text {min }}^{\top}\right)^{*}=A^{*}$. If a normal system $\left\{B_{j}\right\}_{j=0}^{m-1}$ is chosen to be formally self-adjoint, that is, $\hat{A}_{B}=\left(\widehat{A}_{B}\right)^{*}$, then a system $\left\{C_{j}\right\}_{j=0}^{m-1}$ can be chosen formally self-adjoint too. In this case $B_{j}^{\top}=B_{j}$ and $C_{j}^{\top}=C_{j}$, and the Green's formula (2.3) holds with $B_{j}^{\top}=B_{j}$ and $C_{j}^{\top}=C_{j}$. Moreover, in this case, $\mu_{j}=\operatorname{ord}\left(C_{j}\right)=\operatorname{ord}\left(C_{j}^{\top}\right)=2 m-1-m_{j}$. It follows that $\Delta_{\partial \Omega, 1, \mu}=\Delta_{\partial \Omega, 1, m}$. Hence, Proposition 2.7 yields the following result:

Proposition 3.1. Let $\mathcal{A}$ be a formally symmetric elliptic differential expression and assume that $\widehat{A}_{B}$ and $\widehat{A}_{C}$ are self-adjoint. In addition, assume the conditions of Propositon 2.7 are satisfied. Then:

(i) $\Pi=\left\{\mathcal{H}_{\partial \Omega}, \Gamma_{0}, \Gamma_{1}\right\}$ with $\mathcal{H}_{\partial \Omega}=\Pi_{j=0}^{m-1} L^{2}(\partial \Omega)$, and $\Gamma_{\Omega, 0}, \Gamma_{\Omega, 1}$ defined by (2.5), forms a boundary triple for the operator $A^{*}$. In particular, the following Green's formula holds

$$
\left(A_{\max } u, v\right)_{L^{2}(\Omega)}-\left(u, A_{\max } v\right)_{L^{2}(\Omega)}=\left(\Gamma_{\Omega, 1} u, \Gamma_{\Omega, 0} v\right)_{\mathcal{H}_{\partial \Omega}}-\left(\Gamma_{\Omega, 0} u, \Gamma_{\Omega, 1} v\right)_{\mathcal{H}_{\partial \Omega}}, \quad u, v \in D\left(A_{\max }\right) .
$$


(ii) The corresponding Weyl-Titchmarsh operator is given by $M_{\Omega, \Pi}(z)=\left(-\Delta_{\partial \Omega, 1, m}\right)(\Lambda(z)-\Lambda(0))\left(-\Delta_{\partial \Omega, 1, m}\right)$.

For any self-adjoint operator $T=T^{*} \in \mathcal{C}(\mathfrak{H})$ with associated family of spectral projections $E_{T}(\cdot)$, we set $\kappa_{(\alpha, \beta)}(T)=\operatorname{dim}\left(E_{T}((\alpha, \beta)) \mathfrak{H}\right),-\infty \leq \alpha<\beta$ (these numbers may of course be infinite).

Theorem 3.2. Suppose that $A>0$ is a positive definite elliptic operator, and $\Pi=\left\{\mathcal{H}_{\partial \Omega}, \Gamma_{0}, \Gamma_{1}\right\}$ is the boundary triple for $A^{*}$ in Proposition 3.1 with $A_{0}:=A^{*} \uparrow \operatorname{ker}\left(\Gamma_{0}\right)=\widehat{A}_{\gamma_{D}}$, the Dirichlet realization of $A$. Assume also that the operator $\widehat{A}_{C}>0$ is positive definite, $0 \in \rho\left(\widehat{A}_{C}\right)$. Let $K$ be a densely defined (not necessarily closed) symmetric operator in $\mathcal{H}_{\partial \Omega}$ and $A_{K}$ the corresponding extension defined by (2.7). Then:

(i) The Calderon operator $\Lambda(0)$ is self-adjoint and negative definite, $\Lambda(0)<0$.

(ii) If $K$ is $\Lambda(0)$-bounded with bound strictly less than one, then $A_{K}$ is symmetric (but not necessarily closed). If in addition, $\operatorname{ran}(K-\Lambda(0))$ is closed, then so is $A_{K}$, that is, $A_{K} \in \mathcal{C}\left(L^{2}(\Omega)\right)$.

(iii) If $K$ is $\Lambda(0)$-compact and self-adjoint, then $A_{K}$ is self-adjoint $A_{K}=\left(A_{K}\right)^{*}, \kappa_{(-\infty, 0)}\left(A_{K}\right)<\infty$, and

$$
\kappa_{(-\infty, 0)}\left(A_{K}\right)=\kappa_{(-\infty, 0)}\left(I_{L^{2}(\partial \Omega)}+(-\Lambda(0))^{-1 / 2} K(-\Lambda(0))^{-1 / 2}\right) .
$$

(iv) If $K$ is $\Lambda(0)$-compact and sectorial (resp., $m$-sectorial) with vertex $\zeta$ and semi-angle $\omega \in[0, \pi / 2)$, then $A_{K}$ is sectorial (resp., $m$-sectorial) with vertex $\zeta$ and semi-angle $\omega$ too.

Proposition 3.3. Let $\mathcal{A}$ be formally self-adjoint and assume the conditions of Proposition 3.1. Assume also that $A_{K}=\left(A_{K}\right)^{*}$ is a self-adjoint extension of the form $(2.7)$ with $K \in \mathcal{C}(\mathcal{H})$. Then the absolutely continuous parts $A_{K, \mathrm{ac}}$ and $\widehat{A}_{B, \mathrm{ac}}$ of $A_{K}$ and $\widehat{A}_{B}$, respectively, are unitarily equivalent. In particular, $\sigma_{\mathrm{ac}}\left(A_{K}\right)=\sigma_{\mathrm{ac}}\left(\widehat{A}_{B}\right)$.

Proposition 3.4. Suppose that $A=A_{\min }$ is symmetric, and let $\widehat{A}_{B}=\widehat{A}_{\gamma_{D}}$ be the Dirichlet realization of $A$. Assume the conditions of Theorem 2.12 to be satisfied and that $\widehat{A}_{G}=\left(\widehat{A}_{G}\right)^{*} \in \operatorname{Ell}(A)$ is an elliptic realization of $A$ with $G=\left\{G_{j}\right\}_{0}^{m-1}$. Then the absolutely continuous parts $\widehat{A}_{G, \text { ac }}$ and $\widehat{A}_{\gamma_{D}, \text { ac }}$ of $\widehat{A}_{G}$ and $\widehat{A}_{\gamma_{D}}$, respectively, are unitarily equivalent. In particular, $\sigma_{\mathrm{ac}}\left(\widehat{A}_{G}\right)=\sigma_{\mathrm{ac}}\left(\widehat{A}_{\gamma_{D}}\right)$.

Finally, we turn to eigenvalues in spectral gaps:

Definition 3.5. Let $A$ be a symmetric operator in $\mathcal{H}$. Then $(\alpha, \beta),-\infty<\alpha<\beta<\infty$, is called a gap of $A$ if $\left\|\left(2 A-(\alpha+\beta) I_{\mathcal{H}}\right) f\right\|_{\mathcal{H}} \geqslant(\beta-\alpha)\|f\|_{\mathcal{H}}$ for all $f \in \operatorname{dom}(A)$.

By Corollary 2.11, $\sigma_{\text {ess }}\left(A_{K}\right)=\sigma_{\text {ess }}\left(\widehat{A}_{B}\right)$. Therefore, in the gaps of $\widehat{A}_{B}$, the point spectrum of $A_{K}$ can possibly accumulate at most at the endpoints of the gaps. Next, we actually show that $\sigma_{\mathrm{p}}\left(A_{K}\right)$ cannot accumulate at the left end point of any gap:

Theorem 3.6. Suppose that the conditions of Theorem 3.2 are satisfied, and that $K$ is a symmetric $\Lambda(0)$ compact operator in $\mathcal{H}_{\partial \Omega}$. In addition, let $(\alpha, \beta)$ be a finite gap of $A_{0}=\widehat{A}_{\gamma_{D}}$ and introduce $T_{0}(z)=\Lambda(z)-\Lambda(0)$. Then:

(i) $T(z)=\overline{T_{0}(z)} \in \mathcal{B}_{\infty}\left(\mathcal{H}_{\partial \Omega}\right)$ for all $z \in \rho\left(\widehat{A}_{\gamma_{D}}\right)$.

(ii) There exists $\varepsilon_{0} \in(0,(\beta-\alpha) / 2)$ such that $E_{A_{K}}\left(\left(\alpha, \alpha+\varepsilon_{0}\right)\right)=0$, hence $\kappa_{(\alpha, \beta-\varepsilon)}\left(A_{K}\right)=\operatorname{dim}\left(E_{A_{K}}((\alpha, \beta-\right.$ $\varepsilon)))<\infty$ for any $\varepsilon \in(0, \beta-\alpha)$. Moreover, for any $\varepsilon \in\left(0, \varepsilon_{0}\right)$ the following equality holds (with $\left.\Lambda:=\Lambda(0)\right)$ :

$$
\kappa_{(\alpha, \beta-\varepsilon)}\left(A_{K}\right)=\kappa_{(-\infty, 0)}\left(I_{\mathcal{H} \partial \Omega}+\Lambda^{-1 / 2}(K+T(\beta-\varepsilon)) \Lambda^{-1 / 2}\right)-\kappa_{(-\infty, 0)}\left(I_{\mathcal{H} \partial \Omega}+\Lambda^{-1 / 2}(K+T(\alpha+\varepsilon)) \Lambda^{-1 / 2}\right) .
$$

Remark 3.7. For Robin-type realizations $[\partial u / \partial n-\sigma u] \mid \partial \Omega=0, \sigma \in L^{\infty}(\partial \Omega)$, of Schrödinger operators $-\Delta+q$ on exterior domains $\Omega \subset \mathbb{R}^{3}$, the estimate (2.9) (with $\ell=1$ ) goes back to the pioneering work by Povzner [14]. For Robin realizations $A_{\sigma}$ of a second-order elliptic operator $\mathcal{A}=-\sum_{j, k=1}^{n} \frac{\partial}{\partial x_{j}} a_{j, k}(x) \frac{\partial}{\partial x_{j}}+q(x)$, with $q \geq 1$, and $\sum_{j, k=1}^{n} \xi_{j} a_{j, k}(x) \xi_{k}>0$ for all $\{x, \xi\} \in \Omega \times\left(\mathbb{R}^{n} \backslash\{0\}\right)$,

$$
A_{\sigma}=A_{\max }\left\lceil\operatorname{dom}\left(A_{\sigma}\right), \operatorname{dom}\left(A_{\sigma}\right)=\left\{u \in H^{2}(\Omega) \mid(\partial u / \partial \nu-\sigma u) \uparrow \partial \Omega=0\right\}, \partial / \partial \nu=\sum_{j, k=1}^{n} a_{j, k}(x) \cos \left(n, x_{j}\right) \frac{\partial}{\partial x_{k}},\right.
$$

$\sigma \in L^{\infty}(\partial \Omega)$, the estimate (2.9) was obtained by Birman [5]. Moreover, in [5, Thm. 6.6] it is also proved that $\kappa_{(-\infty, 0)}\left(A_{\sigma}\right)<\infty$. Thus, for $m=1$ and $A_{K}=A_{\sigma}$, equality (3.1) with $K$ being a multiplication operator, $K: u \mapsto \sigma u$, yields a stronger result as it describes the actual number of eigenvalues in the gap $(-\infty, 0)$. 
For positive elliptic realizations $\widehat{A}_{G}$ of a nonnegative elliptic operator $A$ of order $2 m$ in a bounded domain $\Omega \subset \mathbb{R}^{n}$, the estimate (2.9) is implied by a sharp estimate due to Grubb [10, eq. (3.22)].

Detailed proofs of these results will appear in [8].

Acknowledgments. Mark Malamud is indebted to the Department of Mathematics of the University of Missouri, Columbia, MO, USA, for the hospitality extended to him during his month long stay in April/May of 2007 in connection with a Miller Family Scholarship, which enabled this collaboration.

\section{REFERENCES}

[1] S. Agmon, A. Douglis, L. Nirenberg, Estimates near the boundary for solutions of elliptic differential equations satisfying general boundary conditions. I, Commun. Pure Appl. Math. 12, 623-727 (1959).

[2] M. S. Agranovich, Elliptic Boundary Problems, in Partial Differential Equations XI, Encyclop. Math. Sci., Vol. 79 , M. S. Agranovich, Yu. V. Egorov, and M. A. Shubin (eds.), Springer, Berlin, 1997.

[3] R. Beals Non-local boundary value problems for elliptic operators, Amer. J. Math. 87, 315-362 (1965).

[4] Ju. Berezanskii, Expansions in Eigenfunctions of Selfadjoint Operators, Transl. Math. Mongraphs, Vol. 17, Amer. Math. Soc., Providence, R.I., 1968.

[5] M. Sh. Birman Perturbation of continuous spectrum of singular elliptic operator under changes of boundary and boundary conditions, Vestnik Leningradskogo Universiteta, No. 1, 22-55 (1962) (Russian).

[6] B. M. Brown, G. Grubb, and I. G. Wood, $M$-functions for closed extensions of adjoint pairs of operators with applications to elliptic boundary problems, preprint, 2008, arXiv:0803.3630.

[7] V. A. Derkach and M. M. Malamud, Generalized resolvents and the boundary value problems for Hermitian operators with gaps, J. Funct. Anal. 95, 1-95 (1991).

[8] F. Gesztesy and M. M. Malamud, Elliptic Boundary Value Problems, Operator-Valued Weyl-Titchmarsh functions, and the Associated Extension Theory, in preparation.

[9] G. Grubb, A characterization of the non-local boundary value problems associated with an elliptic operator, Ann. Scuola Norm. Sup. Pisa (3), 22, 425-513 (1968).

[10] G. Grubb, Spectral asymptotics for the "soft" self-adjoint extension of a symmetric elliptic differential operator, J. Operator Theory, 10, 9-20 (1983).

[11] J. L. Lions and E. Magenes, Non-Homogeneous Boundary Value Problems and Applications, Vol. I, Springer, Berlin, 1972.

[12] V. E. Lyantse and O. G. Storozh, O. G. Metody teorii neogranichennykh operatorov (Russian). [Methods of the Theory of Unbounded Operators], Naukova Dumka, Kiev, 1983.

[13] M. M. Malamud and V. I. Mogilevskii, Krein type formula for canonical resolvents of dual pairs of linear relations, Meth. Funct. Anal. Topology 8, No. 4, 72-100 (2002).

[14] A. Ya. Povzner, The expansion of arbitrary functions in eigenfunctions of the operator $-\Delta u+c u$, Mat. Sbornik 32, $109-156$ (1953) (Russian); Engl. transl. in Amer. Math. Soc. Transl. (2) 60, 1-49 (1967).

[15] M. L. Višik, On general boundary problems for elliptic differential equations, Trudy Moskov. Mat. Obsc. 1, 187-246 (1952) (Russian); Engl. transl. in Amer. Math. Soc. Transl. (2), 24, 107-172 (1963).

Department of Mathematics, University of Missouri, Columbia, MO 65211, USA

E-mail address: fritz@math.missouri.edu

$U R L:$ http://www.math.missouri.edu/personnel/faculty/gesztesyf.html

Mathematics, Institute of Applied Mathematics and Mechanics, R, Luxemburg str. 74, Donetsk 83114, Ukraine

E-mail address: mmm@telenet.dn.ua 